\title{
Magnetic collimation of relativistic jets: the role of the black hole spin
}

\author{
N. Globus, C. Sauty and V. Cayatte \\ LUTh, Observatoire de Paris, F-92190 Meudon, France \\ email: noemie.globus@obspm.fr
}

\begin{abstract}
An ideal engine for producing ultrarelativistic jets is a rapidly rotating black hole threaded by a magnetic field. Following the $3+1$ decomposion of spacetime of Thorne et al. (1986), we use a local inertial frame of reference attached to an observer comoving with the frame-dragging of the Kerr black hole (ZAMO) to write the GRMHD equations. Assuming $\theta$-self similarity, analytical solutions for jets can be found for which the streamline shape is calculated exactly. Calculating the total energy variation between a non polar streamline and the polar axis, we have extended to the Kerr metric the simple criterion for the magnetic collimation of jets developed by Sauty et al. (1999). We show that the black hole rotation induces a more efficient magnetic collimation of the jet.
\end{abstract}

Keywords. galaxies: jets, ISM: jets and outflows, black hole physics, MHD

\section{Context of the study}

Many mechanisms are invoked to collimate jets. We shall not consider here collimation due to the external medium or by energy dissipation (shocks, reconnection). In Active Galactic Nuclei, it is believed that the disk wind can confine the inner spine jet but this can also be done by magnetized flows. If the jet carries a net current, then it can be magnetically self-confined under the tension associated with the toroidal field lines. A more realistic approach must take into account the contribution of particle pressure as well as the dynamical effects of the Kerr metric. GRMHD probably provides the better description for combining those ingredients.

\section{Meridionally self-similar models}

The steady GRMHD equations consist of a set of coupled, nonlinear, partial differential equations expressing momentum, magnetic and mass flux conservation, together with Ohm's law for a perfect conductor. Under the assumption of axisymmetry, streamlines and magnetic fieldlines are roped on the same flux tube of constant mass and magnetic flux. Four free integrals exist that depends only on the magnetic flux function : the total specific angular momentum carried by the flow and the magnetic field, $L$, the corotation angular velocity of each streamline at the base of the flow, $\Omega$, the ratio of the mass and magnetic fluxes, $\Psi_{A}$ and the Bernoulli energy constant, $\mathcal{E}$. In terms of these integrals and the square of the poloidal Alfvén Mach number, we can express the components of the magnetic field and the bulk velocity. To construct classes of exact solutions, we assume a spherical shape for the Alfvén surface and a dipolar angular dependence for the poloidal velocity and magnetic fields. By means of a separation of the variables in several physical key quantities, the GRMHD equations reduce to a system of ordinary differential equations, suitable for a numerical integration. In meridionally self-similar models, the $\theta$-dependance is prescribed a priori while the radial dependance is derived 

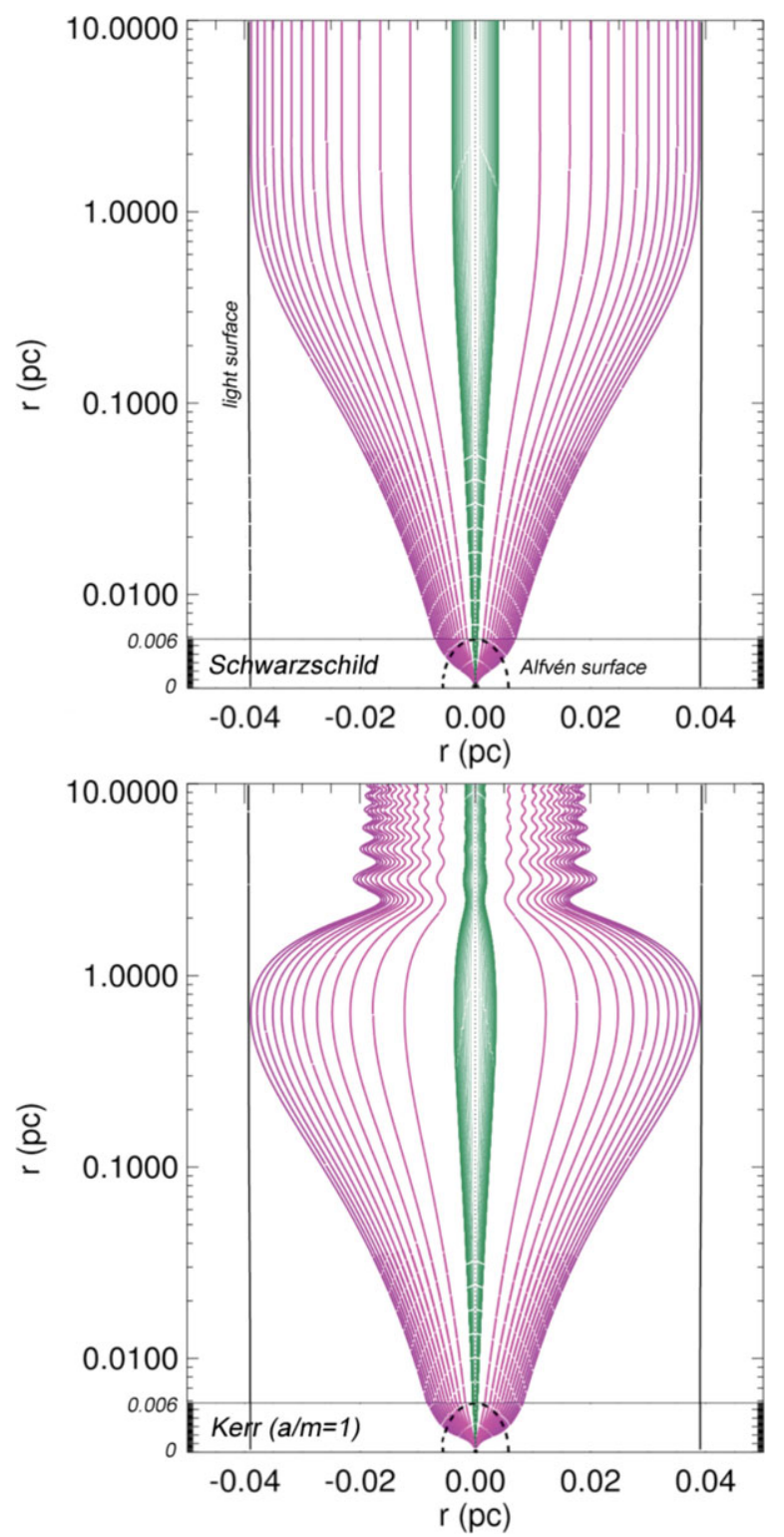

Figure 1. Topology of the solutions in the Schwarzschild metric (upper panel) and in the Kerr metric for a Kerr parameter $a / m=1$ (lower panel).

from the equations. Such a treatment allows to study the physical properties of the outflow close to its rotational axis.

Figure 1 displays two solutions to the GRMHD equations, corresponding to the same physical parameters, except for the spin of the central engine. The lower jet is launched by a Schwarzschild black hole while the upper by a maximally rotating $(a / m=1)$ Kerr black hole. The jet solution in the Schwarzschild case is asymptotically cylindrical while in the Kerr case the solution recollimates at 1 pc, becoming an oscillatory solution with an asymptotic width about half the Schwarzschild one. 


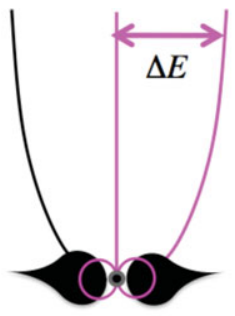

$$
\begin{array}{ll}
\Delta E=\kappa+\varepsilon & \kappa>0: \text { underpressured jet } \\
& \kappa<0: \text { overpressured jet } \\
& \varepsilon>0: \text { efficient magnetic rotator } \\
& \varepsilon<0: \text { inefficient magnetic rotator }
\end{array}
$$

\section{A simple criterion for collimate the flow : $\Delta \mathrm{E}>0$}

\section{The magnetic collimation parameter}

A general criterion for the jet collimation has been established. This criterion was developed successively in the non-relativistic case (Sauty et al., 1999), in the Schwarzschild metric (Meliani et al. (2006)), in the Kerr metric (Globus et al., in prep.). It is based on the variation of energy across the streamlines of the flow :to allow self-confinement, there must be an excess of volumetric energy along a non polar streamline with respect to the axis.

The two solutions have the same $\kappa$ parameter, i.e. the same contribution to collimation of the pressure forces. This implies that the frame-dragging of the Kerr black hole have increased the contribution to collimation of the magnetic forces $\epsilon$. Let express $\epsilon$ at the base of the flow ( subscript $_{o}$ ),

$$
\epsilon=\mathcal{E}_{\text {Rot }, o}+\mathcal{E}_{\text {Poynt. }, o}-\left\|\Delta \mathcal{E}_{\mathrm{G}}^{*}\right\|+L \omega_{o},
$$

where $\mathcal{E}_{\mathrm{Rot}, o}+\mathcal{E}_{\text {Poynt.o }}$ is energy of the magnetic rotator, $\left\|\Delta \mathcal{E}_{\mathrm{G}}^{*}\right\|$ is the gravitational potential which is not compensated by thermal driving (and thus must be supplied by magnetic means), $\omega_{0}$ is the frame dragging potential at the base of the flow. This last term represents the coupling between the orbital angular momentum of the fluid particle $L$ and the frame dragging of the Kerr black hole.

Two new effects due to the Kerr metrics tend to increase $\epsilon$ (Globus et al., in prep.). First $\left\|\Delta \mathcal{E}_{\mathrm{G}}^{*}\right\|$ which is negative, decreases in absolute magnitude in the Kerr metric. Second, the new term $\mathcal{E}_{\mathrm{drag}}=L \omega_{o}$ leads also to an increase of $\epsilon$ because it is positive.

\section{Conclusion}

Compared to the Schwarzschild case, the Kerr metric increases the efficiency of the magnetic rotator to collimate the outflow. Collimation occurs at large distances due to the building of the toroidal magnetic fleld in the super-Alfvénic regime. The rotation of the black hole acts on the collimation since it redistributes the energy of the magnetic rotator and not because it induces directly a strong toroidal magnetic field.

\section{References}

Sauty, C., Tsinganos, K. \& Trussoni, E. 1999, A\&SA, 348, 327

Meliani, Z., Sauty, C., Vlahakis, N., Tsinganos, K. \& Trussoni, E. 2006, A\&AA, 447, 797

Thorne, K. S., Price, R. H. \& McDonald, D. A. 1986, Black Holes: The Membrane Paradigm. 